\title{
PEDIATRICS \\ Hajiyeva N.A. ALAGILLE SYNDROME AS A CHALLENGING CLINICAL CASE IN PEDIATRIC PRACTICE (case report)
}

Azerbaijan State Advanced Training Institute for Doctors, Baku, Azerbaijan

\begin{abstract}
Alagille syndrome is a multisystem, highly variable, autosomal dominant disorder, which can be triggered by spontaneous mutation. This disease primarily affects the liver (chronic cholestasis), heart (most often peripheral pulmonary stenosis), eyes (posterior embryotoxon), face (characteristic features), and skeleton (butterfly vertebrae). The paper presents the clinical case of prolonged jaundice with an increased liver enzymes in infant and no final diagnosis for a long time.
\end{abstract}

KeyWords: Alagille syndrome, chronic cholestasis, ursodeoxycholic acid

\section{INTRODUCTION}

Alagille syndrome (ICD-10:Q44.7; ORPHA:52) - autosomal dominant disorder, which is variably characterized by chronic cholestasis due to paucity of intrahepatic bile ducts, peripheral pulmonary artery stenosis, vertebrae segmentation anomalies, characteristic facies, posterior embryotoxon/anterior segment abnormalities, pigmentary retinopathy, and dysplastic kidneys.

The prevalence of Alagille syndrome (AGS) is approximately $1 / 70,000$. AGS is most commonly due to JAG1 (20p12) gene mutations (AGS type 1), encoding a Notch signaling pathway ligand. AGS type 2 is due to NOTCH2 gene mutations (1p12). The diagnosis is based on the clinical picture and liver biopsy revealing chronic cholestasis and paucity of interlobular bile ducts. Imaging (abdominal ultrasonography, cholangiography) helps to identify biliary anatomy. The clinical symptoms are variable and treatment is non-specific, which causes certain clinical difficulties [1]. We would like to provide a clinical case and share our clinical experience in AGS diagnosis and treatment.

\section{Corresponding Author:}

Corresponding Author:

Nigar Hajiyeva, MD, PhD, Associate Professor, Azerbaijan State Advanced Training Institute for Doctors, Baku, Azerbaijan.E-mail: nigarhajiyeva@yahoo.com

\section{CASE STUDY}

On 21st January, 2015 a 1-year and 2-months-old boy was seen by a pediatrician. The child's parents reported that the boy had been examined by different doctors since birth, had been hospitalized twice and had been consulted by medical professionals abroad. The child's liver enzyme levels, particularly alanine transaminase (ALT) and aspartate transaminase (AST) were constantly increased. The patient received multiple medications, however no symptoms improovement and no final diagnosis till the moment of first admission to our clinic.

The patient's parents are first cousins and there is a second child in the family (his elder sister is healthy).

The child is on adapted formula feeding from birth and was not vaccinated (except vaccinations rendered at birth).

\section{Most recent clinical history and findings:}

On physical examination his weight was measured to be $8 \mathrm{~kg}$ ( 3 percentile), he did not have fever, was neither able to sit, nor to walk, had no teeth, had a prominent forehead and deep-set eyes. There was maculo-papular itching rash on his skin. Neurological examination did not reveal any abnormalities. Skeletomuscular and ophthalmological findings were within normal range. Throat and oral mucosa had no pathological signs. Lymph nodes were not enlarged. Heart auscultation revealed murmur. Respiratory status was normal. Abdomen was soft with normal bowel sounds. Liver was enlarged by 2 centimeters. He was not found to 
have splenomegaly.

In addition, the parents presented the results of previous clinical examinations. The biochemical analysis of blood, taken repeatedly over the year, revealed a steady increase in bilirubin, its fractions, ALT and AST.

Laboratory data on the day of 1-st admission (21st January 2015):

- $\quad$ CBC - slight eosinophilia (4.36), other criteria within normal range;

- $\quad$ CRP, ANA, AMA, total protein, albumin, globulin, calcium - normal.

- Liver enzymes: AST - 202 U/L (Norm - 5-34), ALT - 219 U/L (Norm <55), GGT - 627 U/L (Norm - 12-64);

- Bilirubin: Total - $1.9 \mathrm{mg} / \mathrm{dl}$ (RR 0.2-1.2), conjugated bilirubin - $1.2(\mathrm{RR}<0.5)$, unconjugated bilirubin - 0.7 (RR - 0.2-0.7).

- Echocardiography: pulmonary artery stenosis.

Diagnosing presented certain difficulties. It was obvious that the child had signs of cholestasis with liver parenchyma involvement. The doctor suspected congenital abnormality and its possible association with pulmonary artery stenosis. Following consultations with Professors of Pediatrics from Children's Hospital of Philadelphia and Vienna Medical University, the child was diagnosed with Alagille syndrome (AGS).

This disease was first described by French pediatrician Daniel Alagille in 1969. It is a multisystem, highly variable, autosomal dominant disorder; however the disease can be triggered by spontaneous mutation. The disease is classified as arterio-hepatic dysplasia (disease of the bile duct syndrome) Q 44.7. As a result, lack of the biliary tract in patients causes congenital cholestasis. This disease primarily affects the liver (chronic cholestasis), heart (most often peripheral pulmonary stenosis), eyes (posterior embryotoxon), face (characteristic features), and skeleton (butterfly vertebrae) [2]. AGS has traditionally been diagnosed based on the presence of intrahepatic bile duct paucity on liver biopsy in association with at least three of the major clinical features: chronic cholestasis, cardiac disease, skeletal abnormalities, ocular abnormalities, and charac- teristic facial features.

The patient under examination had three of the signs mentioned above: chronic cholestasis, peripheral pulmonary stenosis and characteristic face features, such as prominent forehead and widely set eyes. He was not found to have embryotoxon and skeleton deformation. AGS is caused by mutation in JAG 1 (20th chromosome) (90\%), in some cases, mutation in NOTCH 2 (1st chromosome). AGS estimation is 1 in 30000 [3].

Since it was impossible to conduct genetic examination of the patient, the diagnosis was established clinically and the treatment process began. The patient was administered ursodeoxycholic acid $15 \mathrm{mg} / \mathrm{kg} /$ day, vitamin D 3000 $\mathrm{U} /$ day and vitamin A $4500 \mathrm{U} /$ day.

After 2.5 months the child was examined once again. He weighed $9 \mathrm{kq}$ ( 3 percentile) and had 6 teeth. The patient could sit, stand, and walk 2-3 steps without assistance. He had no rash. Itching decreased, but did not disappear.

Laboratory data after 2,5 month of treatment (15th April 2015):

- Liver enzymes: AST - $139 \mathrm{U} / \mathrm{L}$ (decreased by 1.5 times), ALT - $102 \mathrm{U} / \mathrm{L}$ (decreased by 2 times), GGT $258 \mathrm{U} / \mathrm{L}$ (decreased by 2.4 times),

- Bilirubin did not changed

- Lipids (cholesterol, triglycerides) and prothrombin levels were slightly elevated.

- Vitamin D level was normal.

Subsequently when the child turned 2 years old in January 2016 his condition slightly worsened: pruritus increased and levels of AST, ALT, GGT doubled, so the parents agreed to perform a liver biopsy.

Biopsy showed slight inflammation activity, slight fibrosis, and yellow bile pigment in the cytoplasm of hepatocytes, focal necrosis, and hyperemia of sinusoids, periportal fiber growth and slight fibrotic dilation in portal areas and moderate mononuclear inflammatory infiltration of cells.

Liver biopsy can strongly support the AGS diagnosis if there is bile duct paucity. However, if the individual meets clinical criteria, then a liver biopsy is not mandatory for 
however in $30 \%$ of cases there is a need for liver transplant $[4,5]$.

\section{Conflict of interests}

There is no conflict of interests.

\section{REFERENCES}

1. http://www.orpha.net/consor/cgibin/OC_Exp.php?Expert=52

2. Hartley, J. L., Gissen, P, Kelly, D.A (2013). Alagille Syndrome and Other Hereditary Causes of Cholestasis. Clin Liver Dis, 17, pp. 280-300.

3. Krantz, I. D, Piccoli, D.A., Spinner, N.B. (1997). Alagille syndrome. J Med Genet, 34, pp.152-157

4. Turnpenny, P.D, Ellard, S. (2012) Alagille syndrome: pathogenesis, diagnosis and management. European Journal of Human Genetics, 20, pp. 251-257.

5. Vajro, P., Ferrante, L., Paolella, J. (2012). Alagille syndrome: An overview. Clinics and Research in Hepatology and Gastroenterology, 36, pp. 275-277.

\section{line therapy for AGS [2].}

\section{Patient treatment:}

Vitamin D and Vitamin A were no longer required, ursodeoxycholic acid was administered at the same dosage and $4 \mathrm{~g} /$ day of Cholestyramine as a bile salt-binding agent and emollients to keep the skin hydrated were additionally introduced into the therapy. As a result, this modified treatment improved pruritus and appetite.

\section{1 year follow-up (April 2016).}

- The child turned 2.5 years.

- His appetite was good.

- He weighed $10 \mathrm{~kg}$ (3 percentile).

- The patient continued to receive ursodeoxycholic acid, and cholestyramine dosage was increased to $8 \mathrm{mg} /$ day.

- Pruritus still persisted.

Further monitoring of this disease is determined by the degree of cholestasis and possible complications. It is also recommended to check fat-soluble vitamin levels twice a year. According to the textbook, the process might stabilize within the period when the child is $4-10$ years old;
Received: $\quad$ 20-Feb. - 2017

Accepted: 20-Jun. - 2017 